\title{
Lessons for leadership and culture when doctors become second victims: a systematic literature review
}

\author{
Donna Willis, ${ }^{1,2}$ Joanna Yarker, ${ }^{2}$ Rachel Lewis ${ }^{2}$
}

${ }^{1}$ People \& Culture, East London NHS Foundation Trust, London, UK

2Business School, Kingston University Kingston Business School, Kingston-Upon-Thames,

\section{Correspondence to} Dr Donna Willis, People \& Culture, East London NHS Foundation Trust, London E1 8DE, UK; d.willis1@nhs.net

Received 14 September 2018 Revised 24 March 2019 Accepted 17 May 2019 Published Online First 24 June 2019
A Check for updates

(c) Author(s) (or their employer(s)) 2019. No commercial re-use. See rights and permissions. Published by BMJ.

To cite: Willis D, Yarker J, Lewis R. BMJ Leader 2019:3:81-91.

\section{ABSTRACT}

This review set out to understand what leaders and organisational cultures can learn about supporting doctors who experience second victim phenomenon; the types, levels and availability of support offered; and the psychological symptoms experienced. A systematic review of keywords 'Medical Error' [MeSH], 'Near Miss', 'Adverse Event', 'Second Victim' and 'Support' was carried out using CINAHL Plus, Medline and Embase Classic and Embase 1947-2017 databases. Results show that poor organisational culture and leadership negatively influences and hinders doctors who make mistakes. Leaders who promote and create environments for open and constructive dialogue following adverse events enable the concept of fallibility and imperfection to be assimilated into new ways of learning. Guilt and fear are the most consistently reported psychological symptoms along with a perception of loss of professional respect and standing. Doctors often carry unresolved trauma for several years causing them to constantly relive an event. Unchecked, this can lead to poor relationships with colleagues and impact greatly on their ability to sleep and performance at work. The review concludes that a prevailing silence, exacerbated by poor organisational culture, inhibits proper disclosure to the first victim, the patient and family. It also impedes a healthy recovery trajectory for the doctor, the second victim. Leaders of organisations have a vital strategic and operational role in creating open, transparent and compassionate cultures where dialogue and understanding takes place for those affected by second victim phenomenon.

\section{INTRODUCTION}

An adverse event describes 'an injury related to medical management, in contrast to complications of disease'. ${ }^{1}$ A 'near miss' describes a 'serious error or mishap that has the potential to cause an adverse event but fails to do so because of chance or because it is intercepted'. ${ }^{1}$ When an adverse event or near miss happens, considerable suffering and psychological distress can be experienced by the health professional. This is known as 'second victim phenomenon'. 'The 'first victim' is the patient and family, and the 'third victim' was latterly described as the healthcare provider or institution. ${ }^{3}$

Spelling out the prevalence of second victim phenomenon is complex and difficult as many incidents go unreported. Clinicians struggle to find understanding from employers, colleagues and response by way of support. ${ }^{4-6}$ Medical error disclosure and reporting is an ethical and professional obligation, ${ }^{7}$ yet paradoxically there is a declared reluctance to disclose an error to the patient and family, known as the 'disclosure gap'. ${ }^{8-10}$ Reluctance to disclose also exists between colleagues ${ }^{11}$ and is particularly prevalent when a serious medical error requires escalation to a medical regulatory body. ${ }^{12}$ Perhaps one explanation lies in the fact that disclosing an error to a patient/family is one of the most challenging conversations that may take place in a doctor's career. ${ }^{13}$ Reported reasons for failure to disclose include issues of self-perception of the clinician's own incompetence ${ }^{7}$ and fear of legal action. $^{14}$

In the UK, $83 \%$ of the UK Royal College of Physicians (RCP) members reported having personally being involved in at least one near miss and/ or adverse event at any point of their career. ${ }^{15}$ Nonetheless many feel inhibited to speak about the debilitating effects that include shame, guilt, fear, panic, shock and humiliation immediately after the event. ${ }^{16}$ Second victims of many clinical professions are reportedly haunted by re-enactments of the adverse event ${ }^{17}$ and worry about colleagues' thoughts or reactions to their error. ${ }^{18}$ Concerns about the error's effect on their career and a sense of clinical incompetence prevail, as well as feelings of internal inadequacy, leading to self-isolation. ${ }^{17}$ Negative outcomes include long-term absenteeism and leaving the profession. ${ }^{19}$ Furthermore in isolated cases, second victim phenomenon has resulted in incidences of suicide, ${ }^{18}$ with a noted increase in UK female healthcare professional suicide rates, higher than the national average. ${ }^{20}$ Among doctors, general practitioners, psychiatrists and trainees are at greater risk of suicide compared with the general population. ${ }^{21}$

To date, three systematic literature reviews have been conducted. The earliest review detailed the response, impact, coping and learning of professionals involved in a medical error. ${ }^{16}$ It cited widespread positive coping strategies, such as changes in an individual's practice and corrective patient safety actions taking place within departments and institutions, as well as negative coping effects on psychological well-being such as shame, guilt fear, panic, shock and humiliation. It also cited the importance and impact of cultural attitudes in the context of error; how trainees are impacted and influenced by medical culture and management of error in the healthcare setting.

The second described the prevalence, impact and individual coping strategies of second victims. ${ }^{22}$ The review reported a high prevalence of the phenomenon and called for organisations to provide explicit support to the clinician and other front line staff post incident. Furthermore it summarised the psychological, physical, behavioural and cognitive symptoms reported by second victims and 
the possible long-term effects, which include burnout and a decreasing quality of life.

The final review, by the same lead author, focused on how healthcare professionals are supported post event. ${ }^{23}$ This paper reported a wide range of supportive actions at individual, organisational, national and international level for the patient, family, healthcare provider and the organisation. Furthermore, it cited that consensus fails to exist on how second victims are best supported and recommended that future research provides international organisational tools in response.

The limitations of the three reviews as they pertain to this paper are that each selects a diverse and broad range of clinical and non-clinical professionals, including patients as participants. Experiencing an adverse event, near miss or medical error is deeply distressing irrespective of profession yet nurses are the most featured participants among the studies cited. This may be explained by the fact that nursing is one of the largest job families: in the UK nurses outnumber doctors on a ratio of 2.4 nurses to every one doctor in the National Health Service (NHS). ${ }^{24}$ While doctors, nurses and other healthcare professionals work closely in the multidisciplinary team, doctors have a different level of responsibility for patient clinical care.

When medical error occurs, culture plays a strong mediating role. The response of 'silence' rather than open disclosure increases the likelihood of doctors becoming second victims. ${ }^{25}$ Culture creates and incubates attitudes that influence a lack of formal support and poor handling of errors by healthcare institutions. ${ }^{16}$ This further explains why doctors are not the focus of many studies and prevents a specific and deeper understanding on how they experience second victim phenomenon and how leadership and culture may influence their recovery trajectory.

Contextually, the recent high profile case of Dr Hadiza BawaGarba exemplifies where many lessons about support, leadership and culture can be learnt. Dr Bawa-Garba was found guilty of manslaughter and gross negligence after the death of a child at a UK NHS Trust. The doctor was denied permission to appeal

\begin{tabular}{|c|c|c|}
\hline Search number & Database & Search history \\
\hline 1 & $\begin{array}{l}\text { Embase Classic and Embase } \\
\text { 1947-2017 }\end{array}$ & $\begin{array}{l}\text { TI Near Miss AND Tx } \\
\text { Support }\end{array}$ \\
\hline 2 & $\begin{array}{l}\text { Embase Classic and Embase } \\
\text { 1947-2017 }\end{array}$ & $\begin{array}{l}\text { TI Second Victim AND Tx } \\
\text { Support }\end{array}$ \\
\hline 3 & $\begin{array}{l}\text { Embase Classic and Embase } \\
\text { 1947-2017 }\end{array}$ & $\begin{array}{l}\text { TI Medical Error [MeSH] } \\
\text { AND Tx Support }\end{array}$ \\
\hline 4 & $\begin{array}{l}\text { Embase Classic and Embase } \\
\text { 1947-2017 }\end{array}$ & $\begin{array}{l}\text { TI Adverse Event AND Tx } \\
\text { Support }\end{array}$ \\
\hline 5 & CINAHL Plus & $\begin{array}{l}\text { TI Second Victim AND Tx } \\
\text { Support }\end{array}$ \\
\hline 6 & CINAHL Plus & $\begin{array}{l}\text { TI Medical Error [MeSH] } \\
\text { AND Tx Support }\end{array}$ \\
\hline 7 & CINAHL Plus & $\begin{array}{l}\text { TI Near Miss AND Tx } \\
\text { Support }\end{array}$ \\
\hline 8 & CINAHL Plus & $\begin{array}{l}\text { TI Adverse Event AND Tx } \\
\text { Support }\end{array}$ \\
\hline 9 & Medline & $\begin{array}{l}\text { TI Second Victim AND Tx } \\
\text { Support }\end{array}$ \\
\hline 10 & Medline & $\begin{array}{l}\text { TI Medical Error [MeSH] } \\
\text { AND Tx Support }\end{array}$ \\
\hline 11 & Medline & $\begin{array}{l}\text { TI Adverse Event AND Tx } \\
\text { Support }\end{array}$ \\
\hline 12 & Medline & $\begin{array}{l}\text { TI Near Miss AND Tx } \\
\text { Support }\end{array}$ \\
\hline
\end{tabular}

against her sentence, suspended by a medical practitioners tribunal for 12 months, then struck off by the General Medical Council and recently reinstated to the medical register following a series of Court appearances. Her treatment has 'rattled' the medical profession and creates the conditions for a revival of a 'blame culture' in the UK NHS ${ }^{26}$ at a time when a talent exodus from the profession increases. ${ }^{27}$

If support for doctors working in the UK NHS following medical error/adverse event is to be improved, leadership must be effective. ${ }^{28}$ It is vital that we also understand, first hand, the experience of doctors who become second victims to enable high quality, safe and compassionate patient care. Quite aside from a legal duty and employer's obligation to provide employee post incident support, the reduced exposure to stress would immensely benefit doctors' well-being.

The focus for this review is on doctors who experience second victim phenomenon and it specifically explores what leaders and organisational cultures can learn about supporting doctors who experience second victim phenomenon; the types, levels and availability of support offered; and the psychological symptoms experienced.

\section{METHOD}

\section{Search strategy}

Three electronic databases (CINAHL Plus, Medline and Embase Classic and Embase 1947-2017) were searched using title keywords 'Medical Error' (MeSH), 'Near Miss', 'Adverse Event' and 'Second Victim' and keyword 'Support' in the text. Table 1 shows the search terms and the twelve separate searches conducted in January 2017, which yielded 849 papers. Searches were limited to English language and no publication date restrictions were made.

After removing duplicates, two reviewers (DW and JY) conducted a title sift: a first examination of the titles of each article for relevancy based on the inclusion/exclusion criteria in table $2 .^{29}$

\section{Table 2 Inclusion/exclusion criteria applied to searched studies}

Papers included Papers excluded

1. Clearly relate to the Second victim. For example, 'Second victim' rapidresponse team helps fellow clinicians recover from trauma'

2. Specifically relate to the doctor or physician when an adverse event, near miss or medical error takes place

3. Worldwide quantitative or qualitative studies

4. Published in English language
1. Relate specifically to a medical or clinical condition rather than the support for the second victim. For example, 'A global survey of adverse event following immunization surveillance systems for pregnant women and their infants'

2. Relate to or focus on the process of 'reporting systems'

3. Relate to liability, litigation or cost of adverse event, near miss or medical error

4. Relate to an adverse event, near miss or medical error that is attributable to a device or procedure that does not involve a human intervention. For example, 'Serious adverse event reporting in a medical device information system'

5. Population are only nurse or healthcare professional

6. Focus on patient safety

7. Unavailable in English language

8. Unavailable from publisher 


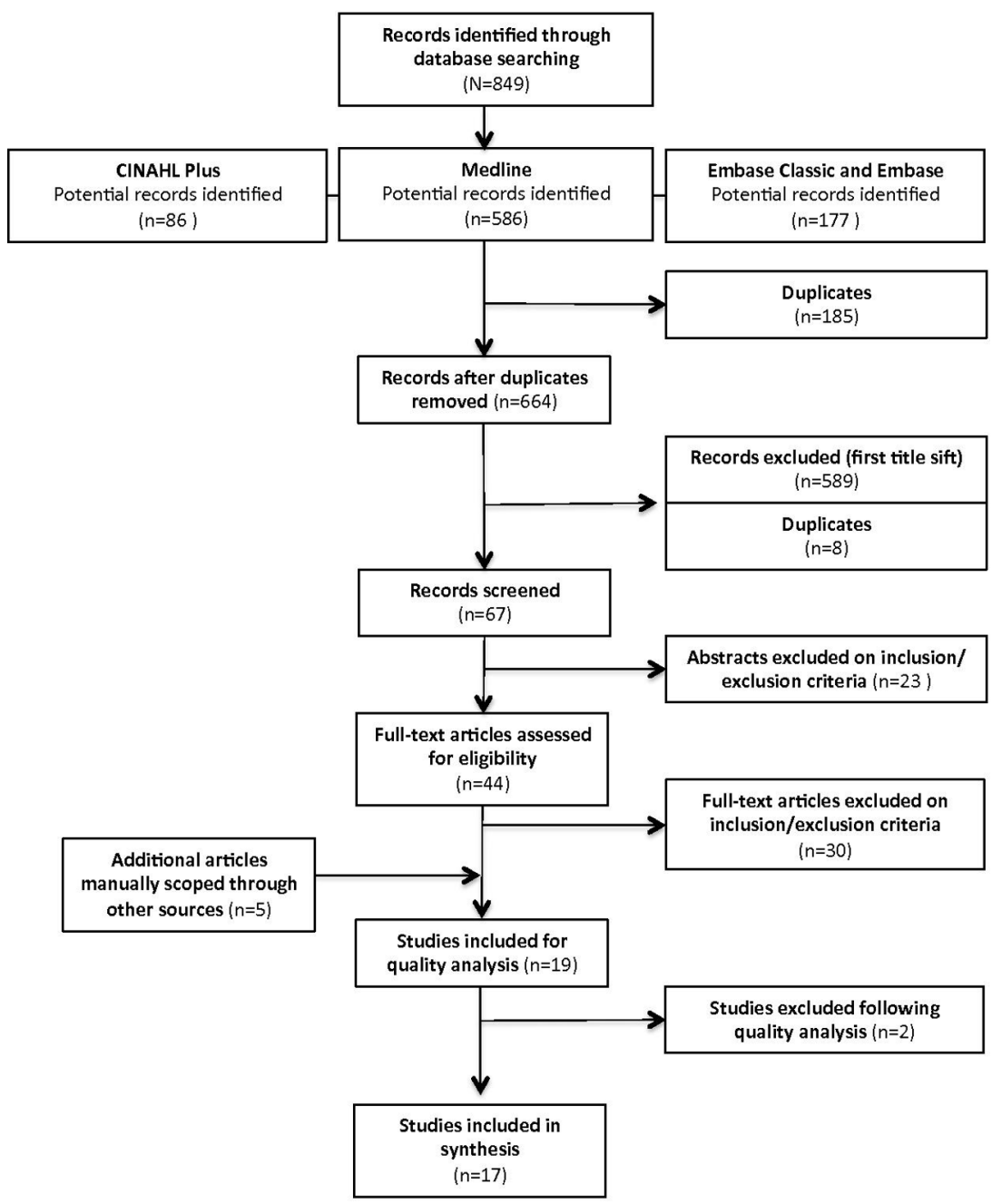

Figure 1 Preferred Reporting Items for Systematic Reviews and Meta-Analyses flow diagram showing search and retrieval process.

\section{Inclusion/exclusion criteria}

Abstracts for the remaining 67 papers were checked against the inclusion/exclusion criteria in table 2 by the first and second reviewers (DW and RL); 23 papers were rejected without disagreement. Full texts of the remaining papers were obtained and applied to the inclusion/exclusion criteria by the same reviewers. The third reviewer (JY) resolved disagreements at this stage.

Using the Matrix method, ${ }^{30} 44$ remaining full text papers were documented and each was evaluated in ascending chronological order using a Review Matrix with columns including: journal identification, purpose, design, participants, measures, findings, key recommendations and limitations. The first and second reviewers rejected 30 papers without disagreement. Five manually scoped papers, agreed by the second and third reviewer (RL and JY), were included at this stage, resulting in 17 papers total. Figure 1 outlines the Preferred Reporting Items for Systematic Reviews and Meta-Analyses flowchart for tracking source documents throughout the review process. ${ }^{31}$

A key part of the systematic review protocol is a quality appraisal. This was conducted using an adapted set of criteria devised from Briner and Denver, ${ }^{32}$ Cohen and Crabtree ${ }^{33}$ and Spencer et al. ${ }^{34}$ For all studies the following criteria were evaluated on a high, high/medium, medium, medium/low or low scale: (i) research quality was ethically carried out; (ii) theoretical and practical importance of the research; (iii) clarity and basis of research question or hypotheses; (iv) appropriateness of sample selection. For qualitative studies the following additional criteria were evaluated using the same scale: (i) extent to which methods were appropriate and rigorous; (ii) clarity and coherence of the research; (iii) consideration given to establishing validity and reliability. For quantitative studies the following additional criteria was evaluated: (iv) appropriateness of data analysis and inferences made. An overall rating was given to each study and studies that scored medium/low. Two studies were rejected at this stage.

The scores for each are summarised in table 3.

\section{RESULTS}

Seventeen studies were included in this review. The primary focus of 11 papers was second victim and the remaining six papers focused on medical error. Participants for all medical error studies were doctors $(n=6)$. Only three $(27 \%)$ of the studies extracted that focused on second victim phenomenon were doctors only. The remaining eight (73\%) consisted of variable numbers of doctors included as part of the participant groups.

Geographically, 53\% $(n=9)$ of the papers were from the USA. ${ }^{1011} 131735-39$ One paper was from the $\mathrm{UK}^{15}$ although one further paper was a joint US/UK study. ${ }^{40}$ The remaining papers were from Europe ${ }^{41-45}$ and Iran. ${ }^{12}$ Finally $65 \%(n=11)$ of the 


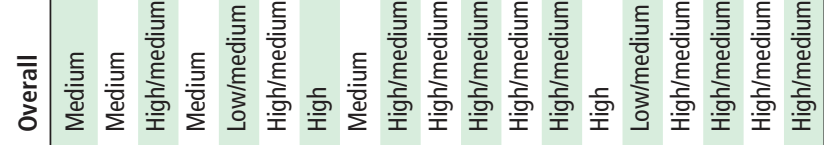

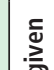

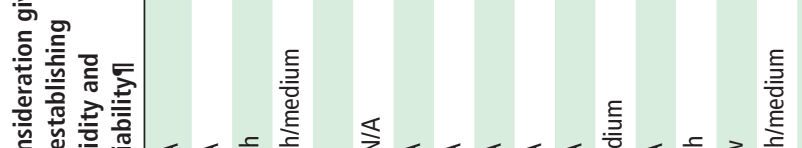

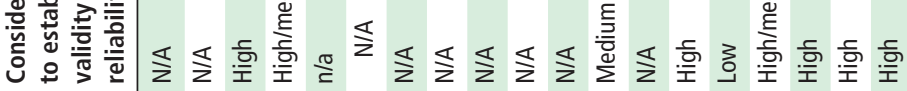

tั

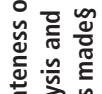

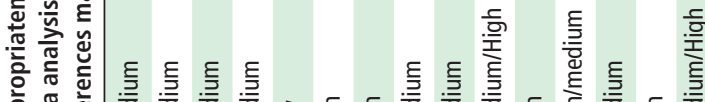

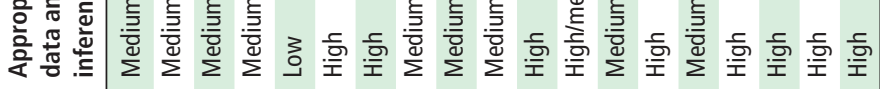

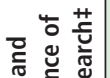

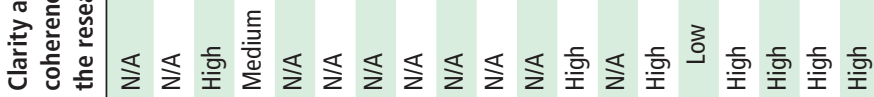

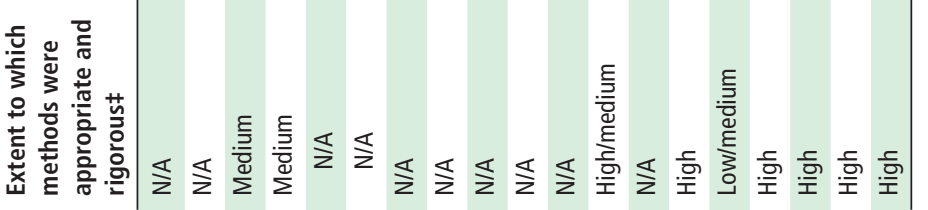

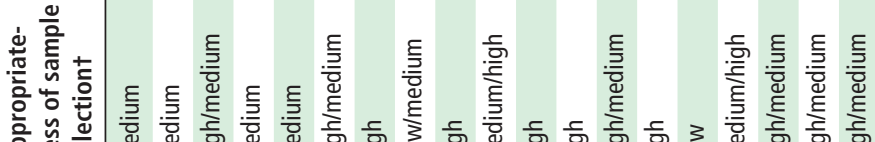

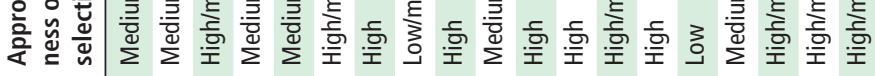
$\frac{n}{\pi}$

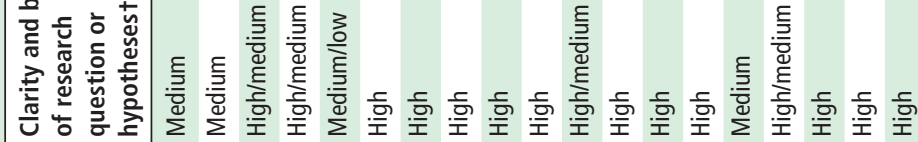

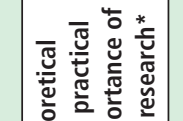

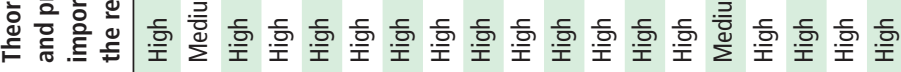

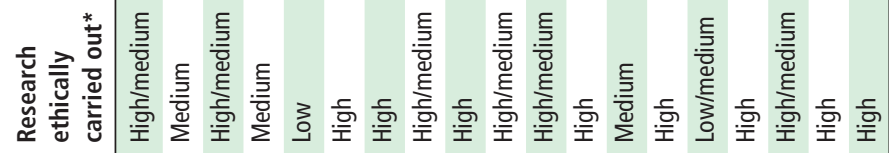

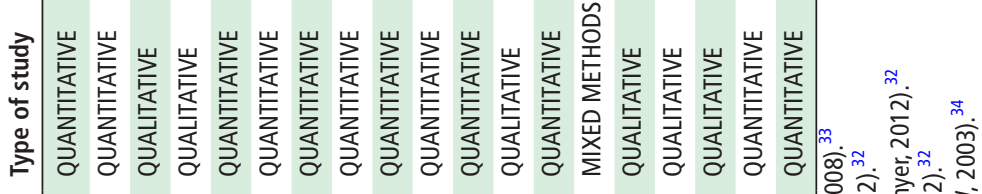

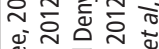


papers utilised a quantitative design, $29 \%(n=5)$ were qualitative and one study was a mixed-method design.

The key characteristics of the papers reviewed can be found in table 4.

\section{Leadership and culture \\ Research question 1: what can leaders and organisational cultures learn about supporting doctors who experience second victim phenomenon?}

Organisational culture is the set of shared, implicit assumptions that members hold which determine how they perceive, think about and react to their environment. ${ }^{46}$ Over $82 \%$ of studies (14 out of 17 papers) adduce that poor organisational culture influences and hinders doctors and other health professionals who make mistakes.. ${ }^{11} 12151736-45$ Culture is implicitly and explicitly set at many levels in healthcare: by commissioners, policy makers, regulators and professional bodies as well as by the organisation and leader's response to an adverse event. The availability and efficacy of institutional support systems directly impacts the doctor who has made a mistake ${ }^{154344}$; however, a Belgian cross-sectional study found that support protocols alone do not influence psychological impact or recovery. Only when support includes retrospective exploration, guidance and forward support was there a positive association with psychological recovery. ${ }^{44}$ Where poor systems and cultures exist, and leaders or peers act as if nothing has happened post error; doctors report stigmatisation and further adverse events occur. ${ }^{39}$ Cultures where mistakes cannot be openly accepted create conditions whereby doctors carry silent and shameful secrets about their mistakes. ${ }^{39} 41$ These are potentially harmful to the doctor and patient alike.

Leaders who offer visible commitment to those affected are seen as most helpful to the doctor's recovery trajectory. ${ }^{36} 4143$ Such leadership is required to facilitate open, honest and transparent discussion to ensure mistakes can be discussed without judgement. ${ }^{12} 151738394243$ Furthermore acknowledgement that medical error is inherent in medicine is important. Blame was described by one study as 'devastating' on the impact and recovery of second victims. ${ }^{44}$ Leaders have a role in reassuring and supporting doctors without blame or minimising the event,${ }^{414}$ instead establishing ways by which doctors can proactively prevent error recurrence. Quality improvement initiatives $^{13}$ along with opportunities to teach others from their experience ${ }^{39} 44$ are reportedly beneficial. Van Gerven et al stipulate that quality improvement needs to go beyond the 'Triple Aim' (improving population health, patient experience and reducing cost) to a 'Quadruple Aim' (the former plus improving the work life of clinicians and staff). Staff and clinicians experiencing stress and burnout impair the ability to achieve the former. ${ }^{44}$

Five studies identify the implementation of a just culture as contributory to patient safety and potential healing for second victims. ${ }^{1136384142}$ A just culture recognises that competent people make mistakes. It also distinguishes between 'error', 'at-risk behaviour' and 'reckless action', enabling appropriate leader responses that hold direct reports to account retributively or restoratively, without being unnecessarily punitive. ${ }^{3647}$ Wisdom and post-traumatic growth formed the conceptual framework of a US study, demonstrating how doctors can use post-traumatic growth to emerge from a serious event with wisdom rather than the usual devastating emotional, cognitive and behavioural effects. ${ }^{39}$ This opens up potential possibilities for leaders to create climates where doctors can find ways to grow from trauma to enable them to continue practising, lead healthy lives and help others to process adverse events.

The 'hidden curriculum' in medicine prevents such growth. It is characterised by a response of silence when errors happen with no opportunity for those affected to deal with the difficult ensuing emotions. ${ }^{11}{ }^{38}$ Leaders' reactions to incidents are of paramount consideration since negative rather than positive role-modelling has a greater influence, particularly for juniors, ${ }^{11}$ particularly in relation to duty of candour. ${ }^{11} 123742$ One study calls for leaders to challenge doctors who display 'dysfunctional' responses or behaviours towards their affected colleagues. ${ }^{11}$ Finally, the doctor's recovery, regardless of their career stage, is impeded when conversations are absent, cruel or ostracised by silence from colleagues and supervisors. ${ }^{173842}$ What is crucial is that leader or peer support, when sought, does not dismiss the seriousness or the reality of the mistake. ${ }^{39}$

\section{Support: types, levels and availability}

Research question 2: what types, levels and availability of support are offered to doctors or physicians who experience medical error and second victim phenomenon?

Irrespective of country, studies mostly describe inadequate and inconsistent levels of available support for second victims. $^{15} 173641-45$ Where support exists, it was found at a number of levels. At an organisational level, doctors along with other colleagues might receive institutionally provided therapeutic support, ${ }^{36}$ and/or 1:1 crisis intervention. ${ }^{3640}$

At team level, team meeting discussions or team debriefing takes place to process the adverse event. ${ }^{36}$ In some healthcare institutions this takes the form of the Morbidity and Mortality (M\&M) Conference, ${ }^{35}$ a peer review of patient care errors with the purpose of learning from such complications or errors to avoid future repetition. ${ }^{48}$ However, the M\&M Conference does not ordinarily focus on the psychological or emotional needs of the doctor and team who have experienced an event and the use of Critical Stress Incident Management and psychological debriefing may help to support second victims against burnout and other maladaptive coping mechanisms more effectively. ${ }^{45}$

The opportunity to talk with trained peers is the most reportedly favoured form of support, but at the same time, dissatisfaction and concerns are held about formal institutional reporting processes and confidentiality breaches. ${ }^{15}{ }^{40}$ Sharing an untoward experience with a 'peer with an ear ${ }^{39}$ is seen as crucial just as long as the colleague is non-judgemental ${ }^{43}$ and maintains confidentiality. ${ }^{40}$ Formal mentors and colleagues from other health professions are also accessed to provide support ${ }^{15}$ as are friends and family. ${ }^{15}$ However, some studies are contradictory about the benefits reporting that doctors find speaking to non-medics about complex medical matters unfulfilling. ${ }^{43}$

Second victim doctors also demonstrate individual means of coping: adapative and maladaptive. A Belgian study found differences between doctors and nurses engagement with problematic medication use, excessive alcohol consumption, workhome interference (WHI), burnout and turnover reactions following a patient safety incident. Doctors reported excessive alcohol consumption and WHI yet lower turnover intentions than nurses. ${ }^{45}$ Conversely, a US study described how approximately one-third of the resident doctors took actions to bring about system changes and improvements as a positive coping mechanism, helping them to deal with the feelings of frustration. ${ }^{35}$ Further individual means of coping might involve seeking faith, prayer and developing self-forgiveness but also writing about their experience, either privately or publicly. ${ }^{38}$ 


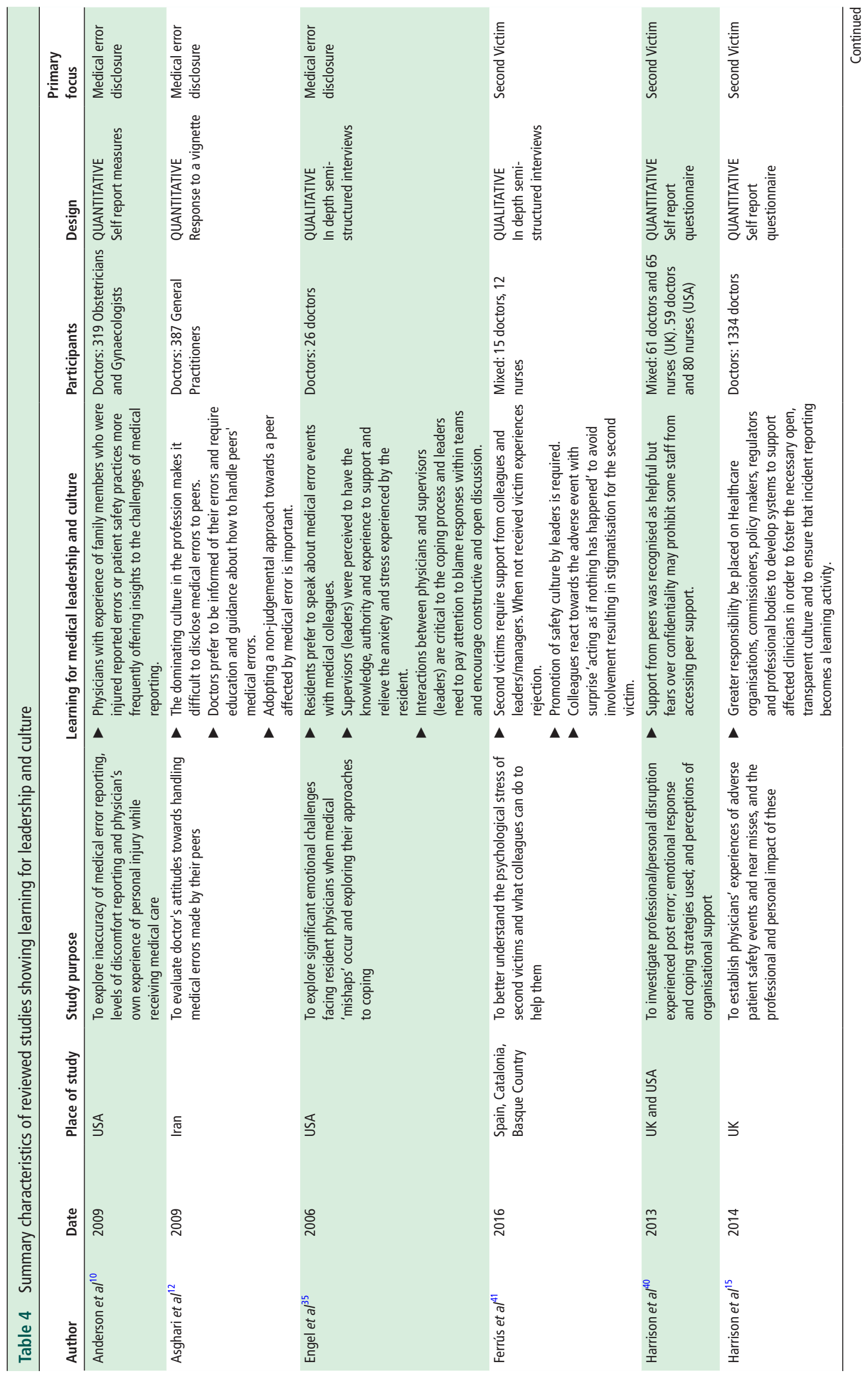




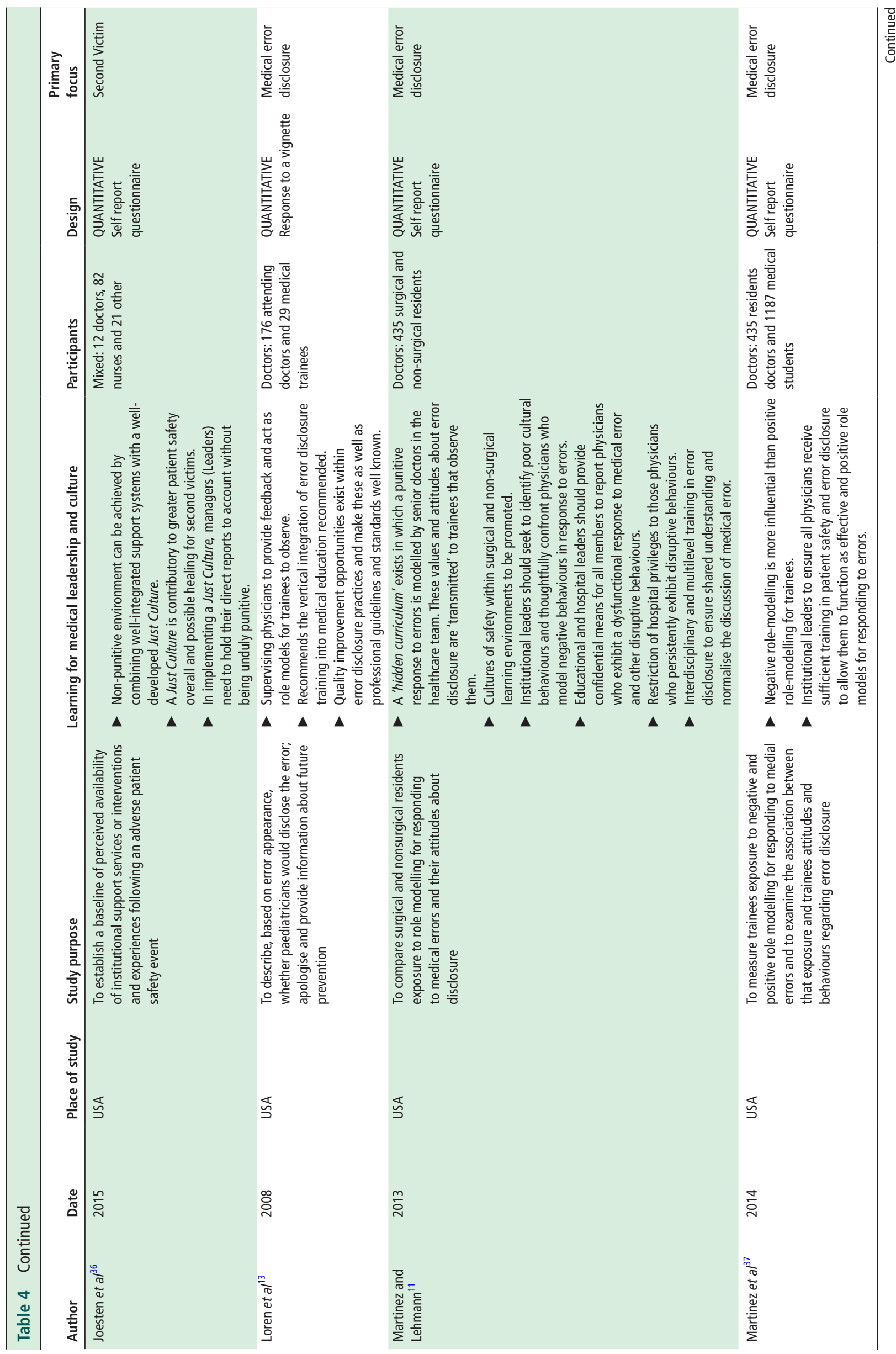




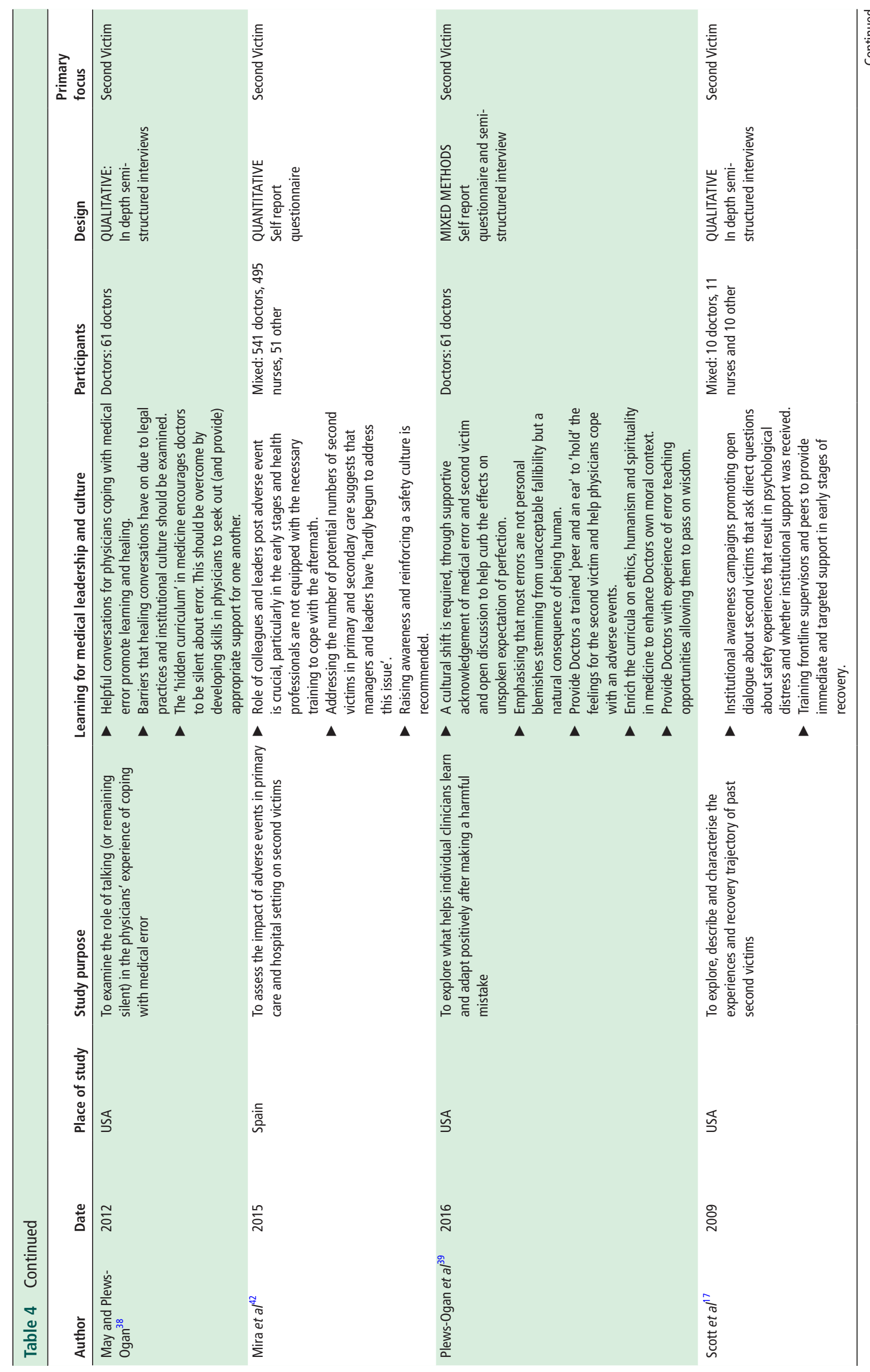




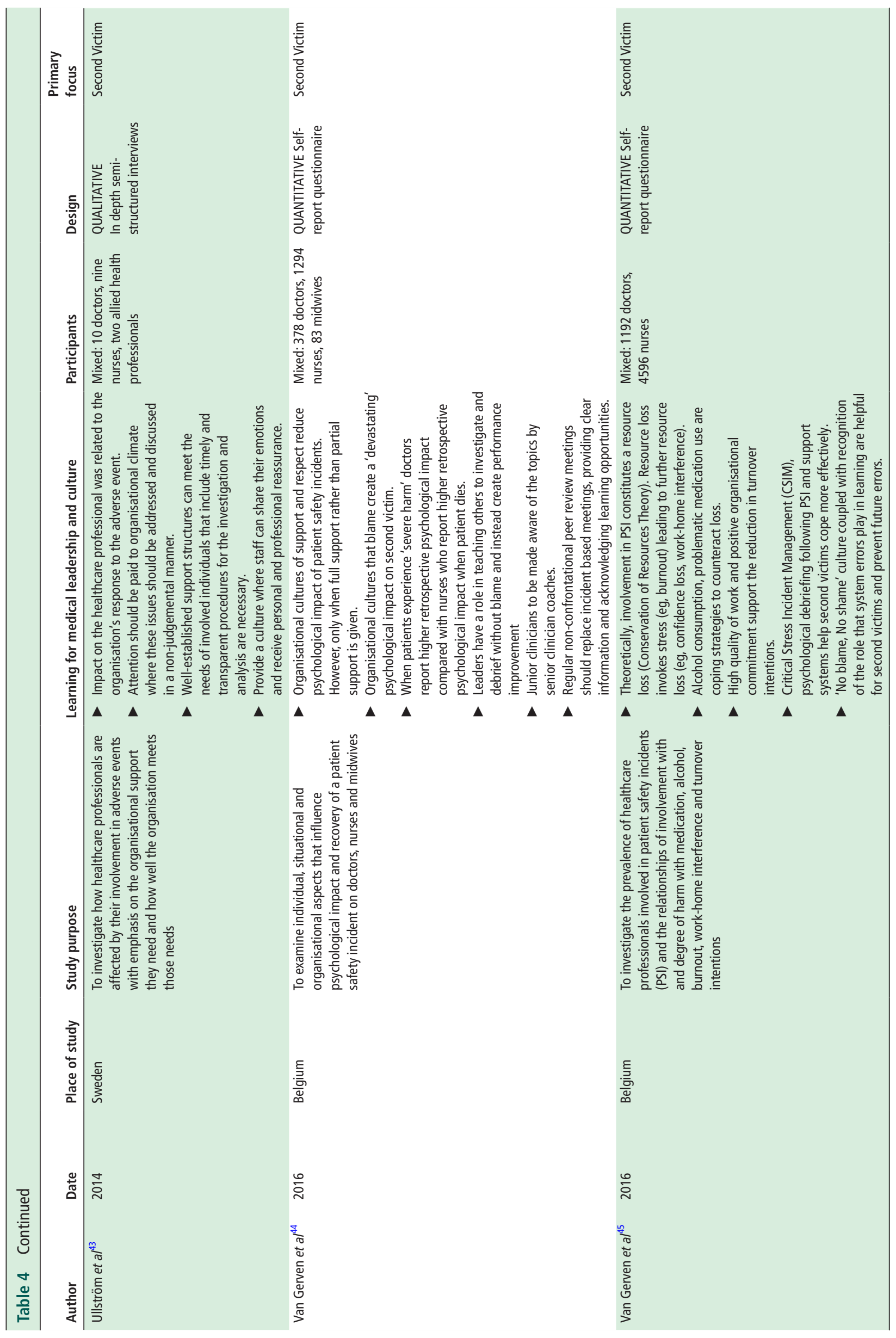




\section{Psychological symptoms}

Research question 3: what are the psychological symptoms experienced by doctors or physicians who experience medical error and second victim phenomenon?

Guilt is the most consistently reported psychological symptom experienced following an adverse event or medical error by the seven studies that specifically addressed psychological symptoms as part of their enquiry. ${ }^{15} 35{ }^{39-43}$ In an attempt to distinguish between doctors and other health professionals, the findings from studies with only doctors as participants ${ }^{15} 3839$ are drawn from to explain psychological symptoms experienced.

First, a UK study of fellows and members of the UK RCP found that nearly $60 \%$ of doctors suffered from difficulty sleeping, potentially contributing a direct detrimental effect on patient safety and the safety culture of UK NHS organisations. ${ }^{15}$ Insomnia, lack of sleep and sleep disturbance is not unique to doctors and reported by three of the seven mixed participant papers that explored psychological symptoms. ${ }^{15} 4243$ Doctors experience more intense reactions when associated with poor patient outcomes and higher levels of personal responsibility. ${ }^{35} 44$ The risk of burnout and problematic medication use was reportedly the same in a large sample. ${ }^{45}$ However, this is not borne out in Harrison et al's UK study where only $27 \%$ of doctors reported negative psychological symptoms or strong feelings of distress. The authors note a response bias whereby doctors strongly psychologically affected or not at all may have chosen not to participate in the member study. ${ }^{15}$

Second, there is a common sense of regret after the incident ${ }^{15}$ disrupting both professional and personal lives. Studies by May and Plews-Ogan and Plews-Ogan et al draw from the same sample of 61 US doctors. ${ }^{38}$ May and Plews-Ogan discuss that doctors are fearful of being 'forever legally vulnerable' and resort to the safe harbour of silence. The authors' findings suggest that helpful conversations promote learning yet, paradoxically, the threat of legal action to both doctor and institution prevents this. ${ }^{38}$ Plews-Ogan et al highlight the doctors' difficulty and struggle with self-forgiveness. The authors explained how the participants mostly 'wrestled' with self-forgiveness as it involved lowering their high standards of perfection or 'letting themselves off the hook'. ${ }^{39}$ Furthermore, the associated shame of the error, coupled by the anger and grief expressed by the patient/family, requires great courage on the part of the doctor to face up to. A subsequent dissociated silent narrative prevails in such socially unacceptable situations and without a supportive and open culture, the doctor carries unresolved trauma alone, in silence and often for many years. ${ }^{39}$ One mixed participant study acknowledged suicidal behaviour among doctors in the introduction. ${ }^{43}$ Excepting this, no other study made reference to or reported findings on suicidal ideation, attempt or completion by doctors who experienced second victim.

Becoming a second victim impacts on the doctor's performance at work. ${ }^{40}$ Post event, over a quarter of UK doctors describe relationships with colleagues as strained and affected. ${ }^{15}$ Worldwide, doctors may feel shunned and rejected by colleagues ${ }^{3641}$ resulting in worry about how others may think about them. ${ }^{3641-43}$ While most studies proclaimed higher levels of negative emotions, four studies cite how doctor's valued their relationships with colleagues more after the incident. This suggests that speaking to colleagues about the error serves as a coping approach to regulate their emotions and reappraise their position. ${ }^{15}{ }^{38-40}$ Harrison et al detailed a number of positive outcomes described by doctors who have experienced an adverse incident and wanted to improve their practice as a result. Just over a fifth had made local improvements, 19\% had made system changes, and just over $8 \%$ had become involved in learning activities. ${ }^{15}$

\section{SUMMARY}

This review has focused on the key learnings for organisational culture and leadership when doctors experience second victim phenomenon following a medical error or adverse event. It also explored the types, levels and availability of support and the psychological symptoms experienced. Seventeen studies, across different healthcare settings and worldwide, were reviewed.

Poor organisational culture and leadership influences and hinders doctors who make mistakes. Evidence from the latest writers on medical culture make a strong and significant case for change since it threatens to inhibit the required learning by pushing those affected and their experiences underground. Doctors are not beyond making mistakes; but when a culture prevents discussion, learning cannot take place and no one, patient, family, peers, the healthcare institution or the doctor, benefits. Leaders who promote and create environments for open and constructive dialogue post incident, rather than blame, enable the concepts of fallibility and imperfection to be assimilated into new ways of learning. Just cultures and those with blameless and supportive leadership contribute towards positive learning climates for all staff to benefit and learn from mistakes. In being 'wisdom' exemplars and through teaching and leading on quality improvement initiatives, doctors have found a means to emerge from error events through positive growth. This is particularly effective for the second victim medic who, with greatest responsibility for the patient, perceives they have much to lose when such lessons cannot be learnt in psychological safety.

The prevalence of second victim phenomenon remains difficult and complex to quantify and report. The reasons for this are various but what preponderates is that the doctor is fearful following an adverse event, perceiving that they have much to lose in respect of their profession and standing. What sets doctors apart from other healthcare professionals is an understandable reluctance to discuss medical error for fear of legislative action. This prevailing silence and consequential lack of support means that doctors carry unresolved trauma alone for many years and this stands in the way of achieving the best patient care and experience.

The findings are therefore mostly concurrent with previous reviews suggesting that little has changed. The strengths of this review is that it draws together and reports on the themes of culture and highlights opportunities for medical leaders and organisations to promote positive culture change in medicine. Furthermore, it attempted deliberately to study doctors as a participant group rather than the multidisciplinary healthcare team and distinguish the psychological symptoms of second victim phenomenon for doctors, what support is available and what they find most helpful.

\section{Limitations and implications for future research}

Papers included in this review consisted of a wide range of studies, comprising of quantitative and qualitative methodological designs, with diverse study aims. This makes comparison difficult. Many papers were cross-sectional and therefore establishing cause and effect is not possible. Furthermore, there is a distinct and notable absence of studies that focus only on doctors who become second victims as participants.

Lastly, the majority of papers for this study are worldwide and mostly from the US where the body of research into second victim phenomenon originates. A void in the literature remains 
in understanding the views of UK doctors where healthcare has a distinctive funding model to other countries where patients and carers, in the main, pay for healthcare. Further research is necessary to develop a more in-depth understanding of how doctors can best recover from second victim phenomenon in the UK.

Contributors DW planned, conducted and reported this review and is responsible for the overall content as guarantor. JY and RL critically reviewed the content of this review and each served as reviewer and advisor, respectively.

Funding The authors have not declared a specific grant for this research from any funding agency in the public, commercial or not-for-profit sectors.

Competing interests None declared.

Patient consent for publication Not required.

Provenance and peer review Not commissioned; externally peer reviewed.

Data sharing statement Data are available upon reasonable request.

\section{REFERENCES}

1 World Health Organization. World Alliance for patient safety: who draft guidelines for adverse event reporting and learning systems: from information to action. Geneva: World Health Organization, 2005.

2 Wu AW. Medical error: the second victim. The doctor who makes the mistake needs help too. BMJ 2000;320:726-7.

3 Denham CR. Trust: the 5 rights of the second victim. J Patient Saf 2007;3:107-19.

4 Gallagher TH, Waterman AD, Ebers AG, et al. Patients' and physicians' attitudes regarding the disclosure of medical errors. JAMA 2003;289:1001-7.

5 Hobgood C, Hevia A, Tamayo-Sarver JH, et al. The influence of the causes and contexts of medical errors on emergency medicine residents' responses to their errors: an exploration. Acad Med 2005:80:758-64.

6 Marmon LM, Heiss K. Improving surgeon wellness: the second victim syndrome and quality of care. Semin Pediatr Surg 2015:24:315-8.

7 Lo B, mistakes D. Disclosing mistakes. In: Lo B, ed. Resolving ethical dilemmas: a guide for clinicians. Baltimore: Lippincott Williams \& Wilkins, 2012

8 Wu AW, Folkman S, McPhee SJ, et al. Do house officers learn from their mistakes? JAMA 1991;265:2089-94.

9 Kaldjian LC, Jones EW, Wu BJ, et al. Disclosing medical errors to patients: attitudes and practices of physicians and trainees. J Gen Intern Med 2007;22:988-96.

10 Anderson B, Stumpf PG, Schulkin J. Medical error reporting, patient safety, and the physician. J Patient Saf 2009;5:176-9.

11 Martinez W, Lehmann LS. The "hidden curriculum" and residents' attitudes about medical error disclosure: comparison of surgical and nonsurgical residents. J Am Coll Surg 2013;217:1145-50.

12 Asghari F, Fotouhi A, Jafarian A. Doctors' views of attitudes towards peer medical error. Qual Saf Health Care 2009;18:209-12.

13 Loren DJ, Klein EJ, Garbutt J, et al. Medical error disclosure among pediatricians: choosing carefully what we might say to parents. Arch Pediatr Adolesc Med 2008:162:922-7.

14 Ghalandarpoorattar SM, Kaviani A, Asghari F. Medical error disclosure: the gap between attitude and practice. Postgrad Med J 2012;88:130-3.

15 Harrison R, Lawton R, Stewart K. Doctors' experiences of adverse events in secondary care: the professional and personal impact. Clin Med 2014:14:585-90.

16 Sirriyeh R, Lawton R, Gardner P, et al. Coping with medical error: a systematic review of papers to assess the effects of involvement in medical errors on healthcare professionals' psychological well-being. BMJ Quality \& Safety 2010;19:e43.

17 Scott SD, Hirschinger LE, Cox KR, et al. The natural history of recovery for the healthcare provider "second victim" after adverse patient events. Qual Saf Health Care 2009;18:325-30

18 Edrees $\mathrm{H}$, Connors $\mathrm{C}$, Paine L, et al. Implementing the rise second victim support programme at the Johns Hopkins Hospital: a case study. BMJ Open 2016;6:e011708.

19 Burlison JD, Quillivan RR, Scott SD. The effects of the second victim phenomenon on work-related outcomes: connecting self-reported caregiver distress to turnover intentions and absenteeism. J Patient Safe 2016. [Epub ahead of print: 2 Nov 2016]. 10.1097/PTS.0000000000000301.

20 De Cates AN, Knott G, Cole-King A, et al. 'Dying to help': female doctor suicide and the NHS workforce crisis. Journal of Holistic Healthcare 2017:14.

21 Kinman G, Teoh $\mathrm{K}$. What could make a difference to the mental health of UK doctors? A review of the research evidence. Society of occupational medicine, 2018.
Available: https://www.som.org.uk/sites/som.org.uk/files/What_could_make_a_ difference_to_the_mental_health_of_UK_doctors_LTF_SOM.pdf [Accessed Nov 2018]

22 Seys D, Scott S, Wu A, et al. Supporting involved health care professionals (second victims) following an adverse health event: a literature review. Int I Nurs Stud 2013;50:678-87.

23 Seys D, Wu AW, Van Gerven E, et al. Health care professionals as second victims after adverse events: a systematic review. Eval Health Prof 2013:36:135-62.

24 NHS Confederation [Internet]. Key statistics on the NHS, 2016. Available: http://www. nhsconfed.org/resources/key-statistics-on-the-nhs [Accessed Feb 2017].

25 Tevlin R, Doherty E, Traynor O. Improving disclosure and management of medical error - an opportunity to transform the surgeons of tomorrow. Surgeon 2013;11:338-43.

26 Cohen D. Back to blame: the Bawa-Garba case and the patient safety agenda. BMJ 2017;359.

27 General Medical Council (GMC). Medical professionalism matters: report and recommendations. [Internet], 2016. Available: http://www.gmc-uk.org/MPM_report. pdf 68646225.pdf [Accessed Jan 2017].

28 Buttigieg SC, West MA, et al. Senior management leadership, social support, job design and stressor-to-strain relationships in hospital practice. I of Health Org and Mgt 2013;27:171-92.

29 Booth A, Sutton A, Papaioannou D. Systematic approaches to a successful literature review. 143. 2 Edn. London: Sage Publications, 2016.

30 Garrard J. Health sciences literature review made easy: The matrix method. $5^{\text {th }} \mathrm{Ed}$. USA: Jones \& Bartlett Learning. Burlington, 2017.

31 Moher D, Liberati A, Tetzlaff J, et al. Preferred reporting items for systematic reviews and meta-analyses: the PRISMA statement. PLoS Med 2009:6:e1000097.

32 Briner RB, Denver D. Systematic review and evidence synthesis as a practice and scholarship tool. In: Rousseau DM, ed. The Oxford Handbook of evidence-based management: companies, classrooms and research. Oxford: Oxford University Press, 2012: 112-29

33 Cohen DJ, Crabtree BF. Evaluative criteria for qualitative research in health care: controversies and recommendations. Ann Fam Med 2008:6:331-9.

34 Spencer L, Ritchie J, Lewis J, et al. Quality in qualitative evaluation: a framework for assessing research evidence. [Internet]. Available: https://www.gov.uk/government/ uploads/system/uploads/attachment_data/file/498321/Quality-in-qualitativeevaulation_tcm6-38739.pdf [Accessed Jul 2017].

35 Engel KG, Rosenthal M, Sutcliffe KM. Residents' responses to medical error: coping, learning, and change. Acad Med 2006;81:86-93.

36 Joesten L, Cipparrone N, Okuno-Jones S, et al. Assessing the perceived level of institutional support for the second victim after a patient safety event. J Patient Saf 2015:11:73-8

37 Martinez W, Hickson GB, Miller BM, et al. Role-modeling and medical error disclosure: a national survey of trainees. Acad Med 2014;89:482-9.

38 May N, Plews-Ogan M. The role of talking (and keeping silent) in physician coping with medical error: a qualitative study. Patient Educ Couns 2012;88:449-54

39 Plews-Ogan M, May N, Owens J, et al. Wisdom in medicine: what helps physicians after a medical error? Acad Med 2016;91:233-41.

40 Harrison $\mathrm{R}$, Lawton R, Perlo J, et al. Emotion and coping in the aftermath of medical error: a cross-country exploration. J Patient Saf 2015;11:28-35.

41 Ferrús L, Silvestre C, Olivera G, et al. Qualitative study about the experiences of colleagues of health professionals involved in an adverse event. J Patient Saf 2016. doi:10.1097/PTS.0000000000000309. [Epub ahead of print: 02 Nov 2016].

42 Mira JJ, Carrillo I, Lorenzo S, et al. The aftermath of adverse events in Spanish primary care and hospital health professionals. BMC Health Serv Res 2015:15:151.

43 Ullström S, Andreen Sachs M, Hansson J, et al. Suffering in silence: a qualitative study of second victims of adverse events. BMJ Qual Saf 2014;23:325-31.

44 Van Gerven E, Bruyneel L, Panella M, et al. Psychological impact and recovery after involvement in a patient safety incident: a repeated measures analysis. BMJ Open 2016:6:e011403.

45 Van Gerven E, Vander Elst T, Vandenbroeck S, et al. Increased risk of burnout for physicians and nurses involved in a patient safety incident. Med Care 2016;54:937-43

46 Schein EH. Organizational culture and leadership. San Francisco: Jossey-Bass, 1992.

47 Dekker SWA, Breakey H. 'Just culture:' Improving safety by achieving substantive, procedural and restorative justice. Saf Sci 2016:85:187-93.

48 Campbell WB. Surgical morbidity and mortality meetings. Ann R Coll Surg Engl 1988:70:363.

49 Rinaldi C, Leigheb F, Vanhaecht K, et al. Becoming a "second victim" in health care: Pathway of recovery after adverse event. Revista de Calidad Asistencial 2016:31(Suppl 2):11-19. 